\title{
BMJ Open Quality Implementation and 1-year follow-up of the cardiovascular ICU standardised handover
}

\author{
Monica Lupei (D) , ${ }^{1}$ Nishkruti Munshi, ${ }^{2}$ Alexander M Kaizer, ${ }^{3}$ Luke Patten, ${ }^{3}$ \\ Joyce Wahr ${ }^{1}$
}

To cite: Lupei M, Munshi N, Kaizer AM, et al. Implementation and 1-year follow-up of the cardiovascular ICU standardised handover. BMJ Open Quality 2021;10:e001063. doi:10.1136/ bmjoq-2020-001063

Received 16 June 2020 Accepted 28 August 2021

\section{Check for updates}

(c) Author(s) (or their employer(s)) 2021. Re-use permitted under CC BY-NC. No commercial re-use. See rights and permissions. Published by BMJ.

${ }^{1}$ Anesthesiology, University of Minnesota School of Medicine Minneapolis, Minnesota, USA

${ }^{2}$ Anesthesiology, Baystate Medical Center, Springfield, Massachusetts, USA

${ }^{3}$ Department of Biostatistics and Informatics, University of Colorado Anschutz Medical Campus, Aurora, Colorado, USA

Correspondence to

Dr Monica Lupei;

lupei001@umn.edu

\section{ABSTRACT}

Background Miscommunication during clinical handover can lead to partial information transfer and healthcare provider dissatisfaction. We hypothesised that a quality improvement project to standardise the cardiovascular intensive care unit (CVICU) handover could improve healthcare provider satisfaction and reduce information omission.

Methods After institutional review board approval, the operating room (OR) to CVICU handover was audited prior, post and 1 year after standardisation implementation. The medical information transferred, healthcare provider participation and satisfaction, and patient outcome data were collected. Additionally, surveys were sent to the $\mathrm{OR}$ and CVICU staff by email.

Results There were 68 handover processes observed. The odds of greater satisfaction with handover for providers were 18 times higher with the process post implementation $(p<0.0001)$ and 26 times higher 1 year after implementation $(p<0.0001)$. There was statistically significant difference between intensive care unit resident presence ( $45 \%$ vs $76 \%$ vs $91 \%, p=0.004$ ), surgical faculty presence ( $10 \%$ vs $36 \%$ vs $45 \%, p=0.034)$ and surgical fellow presence ( $15 \%$ vs $64 \%$ vs $62 \%, p=0.001)$ between the three time periods. More information related to the surgeon ( $5 \%$ vs $52 \%$ vs $27 \%, p=0.002$ ), the medical history ( $65 \%$ vs $96 \%$ vs $91 \%, p=0.014)$ and the cardiopulmonary bypass ( $47 \%$ vs $88 \%$ vs $76 \%, p=0.017$ ) was conveyed. The duration of mechanical ventilation was shorter after implementation ( $2.2 \pm 2.6$ days vs $1.2 \pm 1.9$ days vs $0.5 \pm 1.2$ days, $p=0.026$ ).

Conclusions One year after the OR to CVICU standardised handover implementation, the healthcare provider satisfaction remained increased, more team members participated and the information transfer increased. Although some clinical outcomes improved, further studies are recommended to prove causality.

\section{BACKGROUND}

The introduction of a standardised sign-out was associated with an increase in all key written and oral communication elements. ${ }^{1}$ A study published in 2018 revealed that diagnoses and goals of treatment are not conveyed or retained by intensivists in half of the cases. ${ }^{2}$ An intervention designed using the aviation and Formula One racing model to standardise sign-out from the operating room
(OR) to the paediatric intensive care unit (PICU) lead to improvement of all aspects of the handover. ${ }^{3}$ The standardisation of handover improved communication, teamwork, Intensive Care Unit (ICU) nurses' satisfaction, and it was associated with reduced information omission. ${ }^{4}$ A pretest-post-test experimental design using a standardised handover tool for ICU registered nurses (RNs) sign-out revealed improved 'relationship and communication' and 'satisfaction' scores. ${ }^{5}$ Implementation of a standardised handover in PICU revealed increased provider ratings and improved patients outcomes measured by required respiratory and haemodynamic interventions, decreased antibiotic administration delays and improved analgesia timing. ${ }^{6}$

Before 2017, there was no standardised handoff for cardiac surgery patients arriving from our institution's OR to the cardiovascular intensive care unit (CVICU). The CVICU healthcare providers at our institution openly expressed their concerns about the lack of standardisation of the handover process.

We hypothesised that a quality improvement (QI) project to standardise the handover process in the CVICU, beginning in July 2017, could improve healthcare provider satisfaction and potentially improve the team members' participation and the medical information transfer.

\section{METHODS}

Context and local problem

The handoff for cardiac surgery patients arriving from OR to CVICU at the University of Minnesota Medical Center was an unstructured process before the standardised handover implementation in July 2017. The CVICU at the University of Minnesota is a 17-bed ICU admitting complex patients postcardiac surgery: patients with heart failure or multiple comorbidities post coronary artery 
bypass surgery, valve surgery, implanted left ventricular assist device, heart transplant; patients post-cardiac surgery requiring mechanical support such as Venoarterial Extracorporeal Membrane Oxygenation (VA-ECMO) or percutaneous temporary ventricular support patients. The CVICU team works in a multidisciplinary environment with cardiac surgeons, heart failure cardiologists and interventional cardiology specialists. The cardiac anaesthesiologists bring patients from the OR to the CVICU after cardiac surgery.

Increased dissatisfaction of the CVCU team was related to omissions, distractions during the handover and difficulty finding information after the OR team left. The CVICU nurses wrote reports and brought their concerns up to their supervisors. The CVICU anaesthesiology and surgery residents presented their discomfort to their faculty supervisors.

\section{DESIGN}

The QI project led by one Anesthesiology Critical Care faculty had the support of the Vice Chair for Quality and Safety for the Department of Anesthesiology. The team included cardiac anaesthesiology fellows, anaesthesiology residents, CVICU registered nurses (RNs), certified registered nurse anaesthetists (CRNAs), surgery residents, cardiothoracic surgery faculty and fellows. The multidisciplinary approach for the QI project mirrored the multidisciplinary work environment.

\section{Development of standardised handover}

The CVICU handover form (figure 1) and handover algorithm (figure 2) were designed using the CVICU, anaesthesiology and surgery team input. Multiple versions of the handover form and algorithm were developed and improved. The handover form and algorithm were tested and iterated through revisions 1 month before implementation with input from members of the multidisciplinary team. The CVICU RNs, anaesthesiology residents and fellows, and the CRNAs wanted a detailed report, while the cardiothoracic surgeons preferred a simple version. We opted to maintain the detailed CVICU handover form (figure 1), considering the majority opinion. The algorithm was discussed and improved; we added 1-hour notice before arrival CVICU, start the handover after the patient is on the ICU monitors and is stable clinically, verification of all team members participation before the sign-out.

\section{STRATEGY}

\section{Implementation of standardised handover}

We educated the residents, nurses, anaesthesiologists and surgeons about the standardised handover process

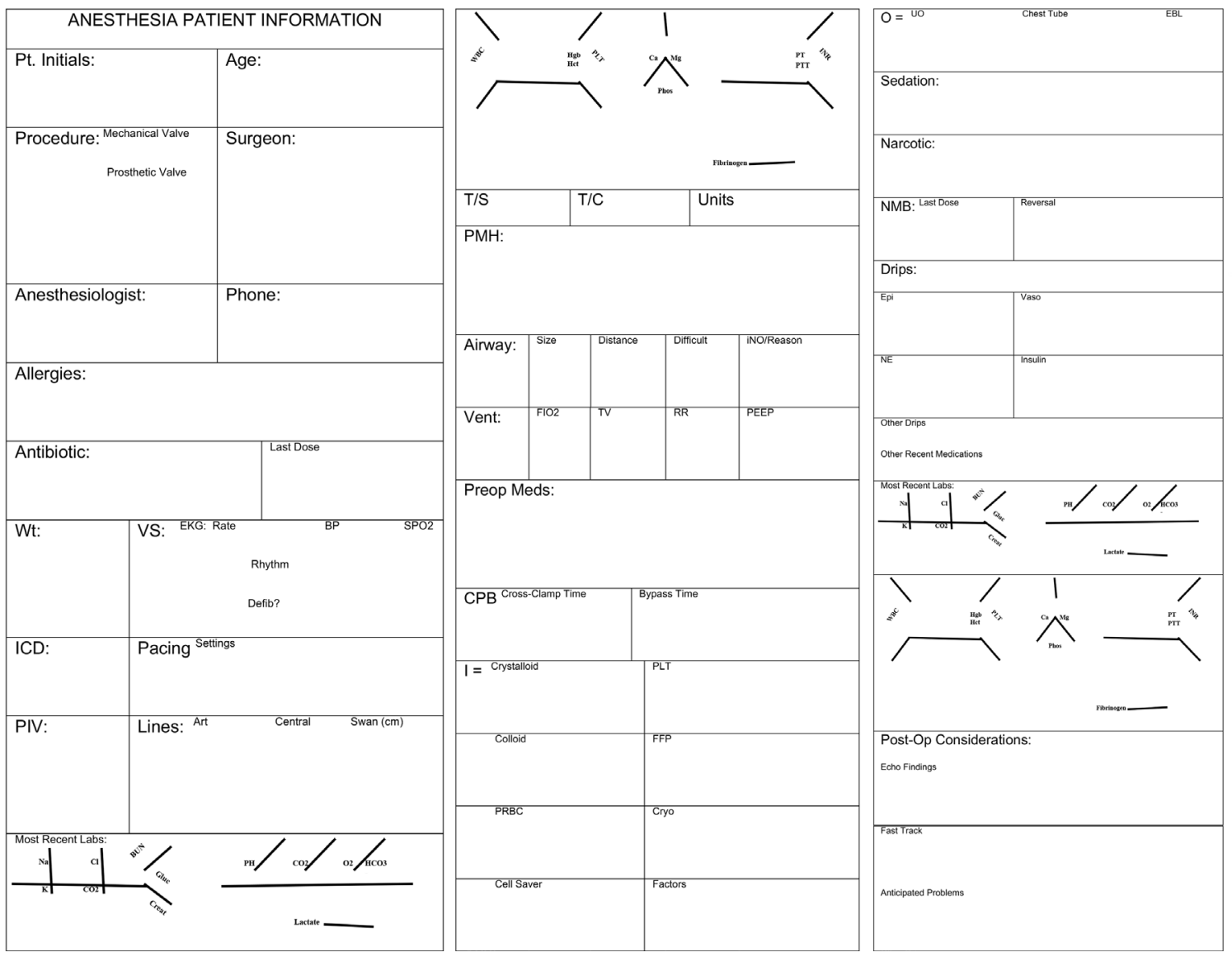

Figure 1 Operating room (OR) to cardiovascular intensive care unit (CVICU) handover form. 


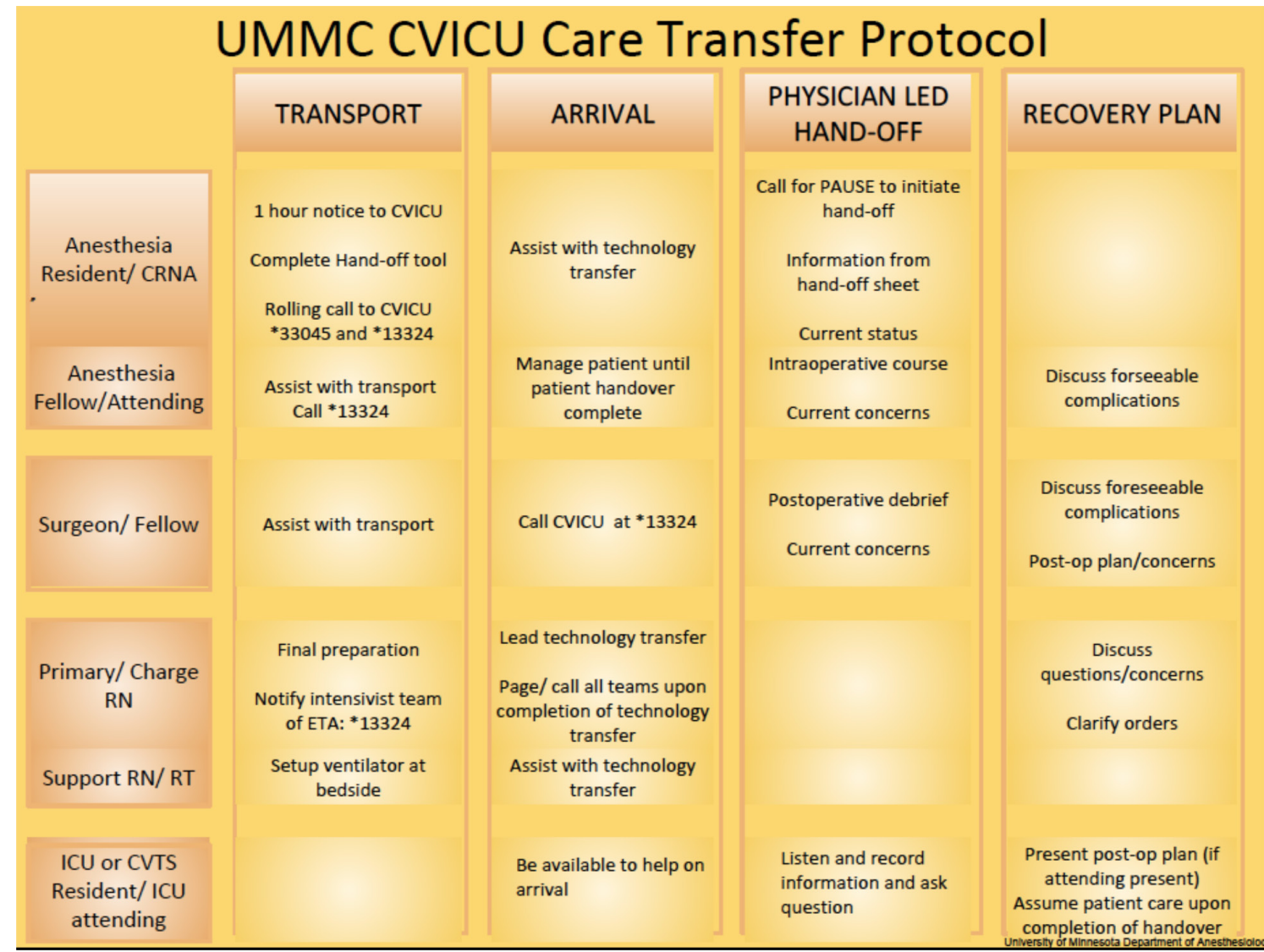

Figure 2 Algorithm of the operating room (OR) to cardiovascular intensive care unit (CVICU) handover. CRNA, certified registered nurse anaesthetist; CVTS, cardiovascular and thoracic surgery; ETA, estimated time of arrival; ICU, intensive care unit; RN, registered nurse.

via email, posters and oral presentations. Additionally, we recruited physicians and nurse champions interested in educating their peers about the new process. Minnesota Health Small Grant Fund financed the QI project. The standardised handover process started in July 2017.

During the handover, each healthcare provider had a clear role; the information transfer started after the ICU monitors were placed and the patient was stable clinically. In the interim, the anaesthesia team was responsible for managing the patient. The sign-out process began only when all the providers were free of tasks and listened to each other. Only one person talked at a time. Each provider had the chance to ask questions at the end of the standardised sign-out.

\section{MEASUREMENTS}

After the institutional review board approval, surveys were sent by email to the multidisciplinary team to assess satisfaction with the handover process before the standardised handover implementation, post implementation and 1 year after implementation. The surveys were concise and contained four key questions:

1. Are you satisfied with the way handoffs are conducted when transitioning patient care from the OR team to the CVICU team?
2. Was the critical information transferred clearly and completely?

3. Did the current handoff negatively affect patient care?

4. Were the key individuals in managing the patient postoperatively missing during the handoff?

Four options were available to choose from in response: completely agree, somewhat agree, somewhat disagree, completely disagree.

The OR to CVICU handoff was audited before, for the first 4 months and 1 year after implementing the standardised handover. Our trained research associates randomly observed the CVICU process before (June 2017), post implementation (July-October 2017) and 1 year after implementation (May-August 2018). The observations were random based on research associates' availability. The anaesthesiology department research associates trained in collecting without interfering with the clinical work; they presented to the CVICU at variable times and days. The clinical team was unaware of their intention to come. The research associates observed the OR to CVICU handover; they collected data about the medical information transferred using the handover form (figure 1). Data collected included the type of procedure, medical history, vital signs, vascular access, allergies, airway details, fluids administered, most recent laboratory results, cardiopulmonary details, anaesthetic 
medications and vasoactive infusions running (figure 1). We also recorded the healthcare provider participation during the handoff.

Following the CVICU handover observation, our research associates collected patients' outcome data from the electronic medical record after the CVICU discharge: mechanical ventilation duration, vasopressor, inotrope treatment duration and ICU length of stay.

\section{EFFECTIVE AIMS}

Our main goal targeted to improve healthcare provider satisfaction. Additionally, we intended to increase team members' participation and the information transferred.

\section{STATISTICAL ANALYSIS}

Data analysis included analysis of variance tests and post hoc t-tests for pairwise comparisons using the Bonferroni correction for continuous outcomes and Fisher's exact tests for binary and categorical outcomes. We compared ORs for the level of satisfaction from questionnaires between periods using univariate ordinal logistic regression models. $\mathrm{P}$ values for independent tests are not adjusted for multiple comparisons and should be interpreted cautiously. All analyses were completed with $\mathrm{R}$ V.3.5.1 (13) and SAS V.9.4.

\section{RESULTS}

We sent the survey to 134 healthcare providers. Before the CVICU handoff implementation, 59 (44\%) healthcare providers responded to the satisfaction survey, $21(16 \%)$ immediately after and $33(25 \%)$ 1 year after the standardised handover implementation. The odds of greater satisfaction with handoffs for healthcare providers were 18 times higher with the process post implementation (95\% CI (4.3 to 75.1), $\mathrm{p}<0.0001$ ) and 26 times higher 1 year after handover implementation compared with the pre-implementation period $(95 \% \mathrm{CI}$ (7.4 to 91.3), $\mathrm{p}<0.0001$ ). The odds that the caregivers agree that the information is transferred clearly and completely during the OR to CVICU handover was 13.5 times higher immediately post-implementation $(95 \% \mathrm{CI}$ (3.9 to 46.6), $\mathrm{p}<0.0001$ ) and 16 times higher 1 year after implementation as compared with pre-implementation (95\% CI (5.2 to 51.1), $\mathrm{p}<0.0001)$. Compared with the pre-implementation period, healthcare providers in the post implementation period are 21 times more likely to disagree that the handoff has a negative effect on patient care $(95 \% \mathrm{CI}(6.2$ to 70.0$), \mathrm{p}<0.0001)$ and 17 times more likely 1 year after implementation to disagree that the handoff has a negative effect on patient care (95\% CI (6.3 to 47.7$), \mathrm{p}<0.0001)$. The odds that the caregivers disagree that key individuals are missing during the handoff are 4 times higher for the post implementation (95\% CI (1.4 to 10.6), $\mathrm{p}=0.011$ ) and 6 times higher for the 1-year post implementation (95\% CI (2.4 to 15.8), p<0.0001) compared with the pre-implementation period. Figure 3 illustrates the satisfaction survey responses.

Around 40 post-cardiac surgery patients are admitted per month in CVICU in our institution. We observed 68 handover processes: 20 before, 26 post and 22 at 1 year after implementation. There was a statistically significant difference between ICU resident, surgery faculty and surgery fellow presence before, after and 1 year after the handover implementation (table 1). A similar percentage of information regarding the type of procedure, the vital signs, the vascular access, the airway details, the fluids administered, the most recent laboratory results, the sedation and the vasoactive infusions was transferred before and after the standardised handover implementation (table 1, p >0.05). More information was conveyed in the first 4 months after and 1 year after the standardised sign-out implementation regarding the medical history, the cardiopulmonary bypass, the allergies and the surgeon involved compared with pre-implementation (table 1, p<0.05).

From a clinical standpoint, the duration of mechanical ventilation was shorter after handover implementation (table 2). We observed a trend towards shorter vasopressor and inotrope treatment duration and reduced ICU length of stay at 1 year (table 2).

\section{DISCUSSIONS}

Implementing a standardised handover for cardiac surgery patients from OR to CVICU at our academic institution was a successful QI project associated with increased healthcare provider satisfaction, increased team members' participation and fewer omissions during information transfer. The results were consistent and durable 1 year after the standardised handover implementation. Additionally, we observed better clinical patient outcomes after the implementation of the standardised handover in CVICU, although we cannot demonstrate causality.

Handoffs between clinical teams can be the source of healthcare provider dissatisfaction. Our preimplementation survey showed that most responders were not satisfied with how the handoffs were conducted, and perceived that key individuals were missing when transferring patient care from OR to CVICU (figure 3). Similarly, a qualitative interview conducted on healthcare professionals and published in 2010 revealed that the handover process is informal, unstructured with incomplete information transfer. ${ }^{7}$ Resembling our study, implementing a pilot handoff protocol from the OR to the cardiac intensive care unit decreased the risk of missed information and increased the ICU nurses' satisfaction. ${ }^{8}$ The implementation of a bundled handover increased healthcare provider satisfaction and the perception of increased efficacy and quality of handoff from the OR to ICU. ${ }^{7}$ While the positive subjective perception might have played a role in our survey results, a standardised multidisciplinary OR to ICU handover was associated with increased healthcare provider satisfaction without an 

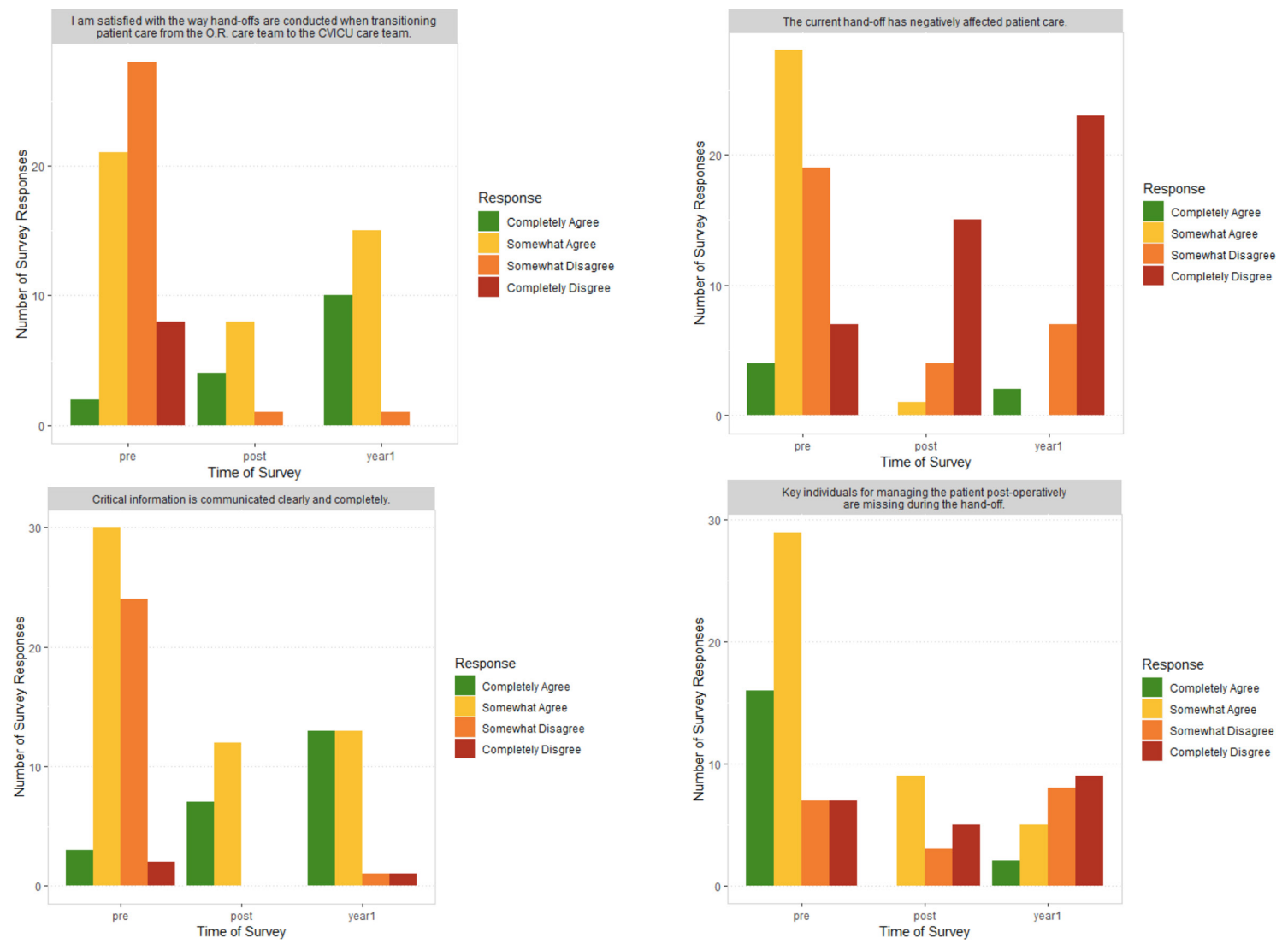

Figure 3 Pre, post and 1 year after implementation survey results.

\begin{tabular}{|c|c|c|c|c|}
\hline Parameter & $\begin{array}{l}\text { Prior to } \\
\text { implementation } \\
(n=20)\end{array}$ & $\begin{array}{l}\text { Post } \\
\text { implementation } \\
(\mathrm{n}=26)\end{array}$ & $\begin{array}{l}1 \text { year after } \\
\text { implementation } \\
(n=22)\end{array}$ & $P$ value \\
\hline ICU resident presence & $45 \%$ & $76 \%$ & $91 \%$ & 0.004 \\
\hline ICU faculty presence & $25 \%$ & $60 \%$ & $41 \%$ & 0.063 \\
\hline Anesthesiology resident or CRNA presence & $90 \%$ & $100 \%$ & $91 \%$ & 0.250 \\
\hline Anaesthesiologist presence & $70 \%$ & $84 \%$ & $73 \%$ & 0.518 \\
\hline Surgical faculty presence & $10 \%$ & $36 \%$ & $45 \%$ & 0.034 \\
\hline Surgical fellow presence & $15 \%$ & $64 \%$ & $62 \%$ & 0.001 \\
\hline Type of procedure & $85 \%$ & $84 \%$ & $91 \%$ & 0.819 \\
\hline Vital signs & $75 \%$ & $76 \%$ & $77 \%$ & 1.000 \\
\hline Vascular access & $80 \%$ & $84 \%$ & $95 \%$ & 0.324 \\
\hline Airway details & $70 \%$ & $88 \%$ & $68 \%$ & 0.189 \\
\hline Fluid administered & $95 \%$ & $96 \%$ & $95 \%$ & 1.000 \\
\hline Most recent laboratory results & $80 \%$ & $96 \%$ & $92 \%$ & 0.208 \\
\hline Sedation & $85 \%$ & $96 \%$ & $91 \%$ & 0.424 \\
\hline Vasoactive infusion & $100 \%$ & $92 \%$ & $95 \%$ & 0.770 \\
\hline Surgeon name & $5 \%$ & $52 \%$ & $27 \%$ & 0.002 \\
\hline Allergies & $30 \%$ & $76 \%$ & $36 \%$ & 0.003 \\
\hline Medical history & $65 \%$ & $96 \%$ & $91 \%$ & 0.014 \\
\hline Cardiopulmonary bypass details & $47 \%$ & $88 \%$ & $76 \%$ & 0.017 \\
\hline
\end{tabular}

CRNAs, certified registered nurse anaesthetists; ICU, intensive care unit. 


\begin{tabular}{|c|c|c|c|c|}
\hline Parameter & Prior to implementation & Post implementation & 1 year after implementation & $P$ value \\
\hline Mechanical ventilation duration & $2.2 \pm 2.6$ days & $1.2 \pm 1.9$ days & $0.5 \pm 1.2$ days & 0.026 \\
\hline $\begin{array}{l}\text { Vasopressor and inotrope } \\
\text { treatment }\end{array}$ & $1.7 \pm 2.2$ days & $1.6 \pm 2.3$ days & $0.5 \pm 0.5$ days & 0.073 \\
\hline ICU length of stay & $4.4 \pm 3.2$ days & $5.2 \pm 8.4$ days & $1.7 \pm 1.3$ days & 0.096 \\
\hline
\end{tabular}

Data presented as mean $\pm S D$.

ICU, intensive care unit.

increase in objectively measured handover time. ${ }^{9}$ Using standardised handover was associated with decreased loss of patient information and improved quality of communication when assessed by an immediate survey. ${ }^{10}$ Our survey results 1 year after implementation showed sustained increased satisfaction and the perception that the critical information was communicated clearly and completely (figure 3). Nevertheless, survey results and self-assessment tools are imperfect, and team performance evaluation during handover needs direct observation. ${ }^{11}$ While we recognise that surveys are subjective, teamwork and more informed communication were associated with increased job satisfaction. ${ }^{12} 13$

We assessed the information transfer from OR to CVICU and the use of handover (figure 1) by direct observation. The evaluation and direct observation of postoperative handover in two European hospitals found that the handoff was associated with frequent distractions incomplete transfer of information. ${ }^{14}$ In the immediate postoperative period, the transfer of information was inconsistent in a Canadian hospital; the only information reported $>90 \%$ of the time consisted in the airway details and the type of procedure. ${ }^{15}$ Before the standardised handover implementation in our CVICU, only the vasoactive medications and the fluid administered information were transferred in $>90 \%$ of the time (table 1 ). One year after implementation, our team transferred $>90 \%$ of the information for the type of the procedure, vascular access, most recent laboratory results, sedation and the medical history (table 1). The use of a checklist increased the data transfer in different studies. ${ }^{46-18}$ Overall, we replicated similar findings: increased information transfer. Our study reached statistical significance by transferring information about the medical history, cardiopulmonary bypass, allergies and the surgeon in the 4 months after and 1 year after the handover implementation. We acknowledge possible undetected confounders, including the Hawthorn effect for the temporal association. ${ }^{19}$ However, the sustainability 1 year after implementation pleads for possible standardised handover association with increased information transfer. Implementing of the standardised handover from OR to ICU increased the healthcare provider involvement, ${ }^{20}$ sometimes non-statistically significant. ${ }^{21}$ More physician providers were present after the standardised handover implementation in our institution (table 1), and our results were consistent 1 year after implementation. The observations were random over
4 months at 1 year after implementation, unannounced, unlikely to influence the physicians' behaviour.

There are mixed results in the literature regarding the handover process improvement and clinical outcome. Following the implementation of handover protocol in a neurosurgical ICU, the rate of ventilator weaning increased, and the duration of mechanical ventilation decreased. ${ }^{22}$ One randomised controlled study found that a standardised handover approach was associated with a trend towards a shorter duration of mechanical ventilation without any change in ICU length of stay and reintubation rate. ${ }^{23}$ The standardisation of the handover in PICU was associated with a decreased need for haemodynamic and respiratory intervention in the first 6 hours of ICU admission. ${ }^{6}$ A prospective interventional study (HATRICC) to implement partially structured handoff in two surgical ICUs did not show any change in ICU length of stay or mortality post-intervention. ${ }^{24}$ Of note, interdisciplinary collaboration was associated with improved patient outcomes. ${ }^{2526}$ In light of these inconsistent literature findings, we recognise the possibility of confounders, including the anaesthesia care intraoperatively, sedation protocols, weaning protocols and the patient selection impacting the patient outcomes. We speculate that cohesive teamwork and better understating of the overall clinical plan can improve patient outcomes. That remains to be evaluated in future studies.

\section{Lessons and limitations}

We learnt important lessons. First, a multidisciplinary approach and collaboration is the key to success when introducing a change. Second, there is potential to increase physician participation and responsibility if the standardised handover transforms into a hospital protocol. Third, the handover form can become more convenient in electronic configuration. Fourth, assessing the efficiency and durability past 1 year might add value to the handover process. Fifth, using a validated tool to assess satisfaction could aid the scientific strength of the handover process evaluation.

Our study has several limitations. First, the study was conducted at one institution in the CVICU only. Second, this is a pre-intervention and post-intervention observational study related to a QI project, not a randomised controlled study, unable to establish causality. Third, a limited number of handover processes were 'randomly' observed during the day, according to the availability of the research associates, which creates bias. Fourth, there 
is potential selection bias among survey responders considering the low response rate, and we can speculate the healthcare providers with interest in success have responded. Fifth, the Hawthorne effect of the observers might have changed the team behaviour and increased the adherence to the handover process.

\section{CONCLUSIONS}

One year after the OR to CVICU standardised handover implementation at our institution, we observed increased healthcare provider satisfaction, enhanced physician participation and reduced patient information omissions during handover. Futures studies are warranted to evaluate the effect of the standardised handover process on team behaviour, efficiency and patient outcomes.

Acknowledgements The authors are grateful to Dr Mike Todd, the Vice Chair of Research in the Department of Anesthesiology, the University of Minnesota, for critically reviewing the manuscript and providing feedback.

Contributors ML made substantial contributions to the conception, the planning, the design and the methodology, the conduct, and the data interpretation of the quality improvement project, applied and obtained an internal grant for the quality improvement project, drafted the work and revised it, finally approved the work to be published, agreed to be accountable for all aspects of the work. NM made substantial contributions to the conception, the planning, the conduct and the data interpretation of the quality improvement project, designed and sent the surveys and analysed the survey results, drafted the work and revised it, finally approved the work to be published, agreed to be accountable for all aspects of the work. AMK made substantial contributions to the conception, the methodology, the statistical analysis and the interpretation of the data, drafted the work and revised it, finally approved the work to be published, agreed to be accountable for all aspects of the work. LP made substantial contributions to the conception, the methodology, the statistical analysis and the interpretation of the data, drafted the work and revised it, finally approved the work to be published, agreed to be accountable for all aspects of the work. JW made substantial contributions to the conception, the planning, the design and the methodology, the conduct, and the data interpretation of the quality improvement project, designed the education material for the quality improvement project, drafted the work and revised it, finally approved the work to be published, agreed to be accountable for all aspects of the work.

Funding This quality improvement project was funded by an internal to our institution grant, MN Health Critical Care Small Grant Fund, between August 2017 and July 2019. No grant/award number.

Competing interests None declared.

Patient and public involvement Patients and/or the public were not involved in the design, or conduct, or reporting or dissemination plans of this research.

Patient consent for publication Not required.

Ethics approval The University of Minnesota Institutional Review Board (IRB) approved our study as non-human research (STUDY00000587).

Provenance and peer review Not commissioned; externally peer reviewed.

All data relevant to the study are included in the article.

Open access This is an open access article distributed in accordance with the Creative Commons Attribution Non Commercial (CC BY-NC 4.0) license, which permits others to distribute, remix, adapt, build upon this work non-commercially, and license their derivative works on different terms, provided the original work is properly cited, appropriate credit is given, any changes made indicated, and the use is non-commercial. See: http://creativecommons.org/licenses/by-nc/4.0/.

ORCID iD

Monica Lupei http://orcid.org/0000-0002-8513-4061

\section{REFERENCES}

1 Starmer AJ, Spector ND, Srivastava R, et al. Changes in medical errors after implementation of a handoff program. $N$ Engl J Med 2014;371:1803-12

2 Dutra M, Monteiro MV, Ribeiro KB, et al. Handovers among staff Intensivists: a study of information loss and clinical accuracy to anticipate events. Crit Care Med 2018;46:1717-21.

3 Catchpole KR, de Leval MR, McEwan A, et al. Patient handover from surgery to intensive care: using formula 1 pit-stop and aviation models to improve safety and quality. Paediatr Anaesth 2007:17:470-8

4 Nagpal K, Abboudi M, Manchanda C, et al. Improving postoperative handover: a prospective observational study. Am J Surg 2013;206:494-501

5 Dalky HF, Al-Jaradeen RS, AbuAIRrub RF. Evaluation of the situation, background, assessment, and recommendation handover tool in improving communication and satisfaction among Jordanian nurses working in intensive care units. Dimens Crit Care Nurs 2020;39:339-47.

6 Breuer RK, Taicher B, Turner DA, et al. Standardizing postoperative PICU handovers improves handover metrics and patient outcomes. Pediatr Crit Care Med 2015;16:256-63.

7 Nagpal K, Arora S, Abboudi M, et al. Postoperative handover: problems, pitfalls, and prevention of error. Ann Surg 2010;252:171-6.

8 Petrovic MA, Aboumatar $\mathrm{H}$, Baumgartner WA, et al. Pilot implementation of a perioperative protocol to guide operating roomto-intensive care unit patient handoffs. J Cardiothorac Vasc Anesth 2012;26:11-16.

9 Krimminger D, Sona C, Thomas-Horton E, et al. A multidisciplinary Qi initiative to improve OR-ICU handovers. Am J Nurs 2018;118:48-59.

10 Agarwal HS, Saville BR, Slayton JM, et al. Standardized postoperative handover process improves outcomes in the intensive care unit: a model for operational sustainability and improved team performance*. Crit Care Med 2012;40:2109-15.

11 Weigl M, Heinrich M, Keil J, et al. Team performance during postsurgical patient handovers in paediatric care. Eur J Pediatr 2020;179:587-96.

12 Moore L, Abell CH, Harris K, et al. Rns' perceptions of engagement and communication in the workplace. J Nurs Adm 2020;50:629-34.

13 Höld J, Späth J, Kricheldorff C. What makes them happy? professional care-givers' job satisfaction. Z Gerontol Geriatr 2020;53:655-62.

14 Nagpal K, Abboudi M, Fischler L, et al. Evaluation of postoperative handover using a tool to assess information transfer and teamwork. Ann Surg 2011;253:831-7.

15 Siddiqui N, Arzola C, lqbal M, et al. Deficits in information transfer between anaesthesiologist and postanaesthesia care unit staff: an analysis of patient handover. Eur J Anaesthesiol 2012;29:438-45.

16 Salzwedel C, Mai V, Punke MA, et al. The effect of a checklist on the quality of patient handover from the operating room to the intensive care unit: a randomized controlled trial. J Crit Care 2016;32:170-4.

17 Karakaya A, Moerman AT, Peperstraete $\mathrm{H}$, et al. Implementation of a structured information transfer checklist improves postoperative data transfer after congenital cardiac surgery. Eur J Anaesthesiol 2013;30:764-9.

18 Dixon JL, Stagg HW, Wehbe-Janek H, et al. A standard handoff improves cardiac surgical patient transfer: operating room to intensive care unit. J Healthc Qual 2015;37:22-32.

19 Franke $\mathrm{RH}$, Kaul JD. The Hawthorne experiments: first statistical interpretation. Am Sociol Rev 1978;43:623-43.

20 Mukhopadhyay D, Wiggins-Dohlvik KC, MrDutt MM, et al. Implementation of a standardized handoff protocol for postoperative admissions to the surgical intensive care unit. Am J Surg 2018;215:28-36.

21 Segall N, Bonifacio AS, Barbeito A, et al. Operating Room-to-ICU patient handovers: a multidisciplinary Human-Centered design approach. Jt Comm J Qual Patient Saf 2016;42:400-5.

22 Yang J-G, Zhang J. Improving the postoperative handover process in the intensive care unit of a tertiary teaching hospital. J Clin Nurs 2016;25:1062-72.

23 Parent B, LaGrone LN, Albirair MT, et al. Effect of standardized handoff curriculum on improved clinician preparedness in the intensive care unit: a Stepped-Wedge cluster randomized clinical trial. JAMA Surg 2018;153:464-70.

24 Lane-Fall MB, Pascual JL, Peifer HG, et al. A partially structured postoperative handoff protocol improves communication in 2 mixed surgical intensive care units: findings from the handoffs and transitions in critical care (HATRICC) prospective cohort study. Ann Surg 2020;271:484-93. 
25 Baggs JG, Schmitt MH, Mushlin Al, et al. Association between nursephysician collaboration and patient outcomes in three intensive care units. Crit Care Med 1999;27:1991-8.
26 Freytag J, Stroben F, Hautz WE, et al. Improving patient safety through better teamwork: how effective are different methods of simulation Debriefing? protocol for a pragmatic, prospective and randomised study. BMJ Open 2017;7:e015977. 